\title{
CONHECIMENTO DO CLIMATÉRIO ENTRE USUÁRIAS DA ESTRATÉGIA SAÚDE DA FAMÍLIA
}

\author{
Raquel Clemente Sousa da Silva I \\ Mayara Layane de Souza Joventino I \\ Débora Raquel Soares Trigueiro " \\ *Vagna Cristina Leite da Silva Pereira II
}

\section{RESUMO}

Eventos importantes marcam o processo de vida das mulheres enquanto enfrentam mudanças orgânicas e psíquicas relacionadas aos ciclos vitais, a exemplo do climatério. $O$ objetivo desse estudo é descrever o perfil sociodemográfico de mulheres climatéricas atendidas na Estratégia de Saúde da Família e constatar o conhecimento das entrevistadas a respeito dessa fase. A pesquisa é descritiva com abordagem quantitativa, realizada em unidade de saúde do município de João Pessoa-PB. A população foi constituída por 894 mulheres, no período do climatério, cadastradas na unidade. Foi definida uma amostra de 100 mulheres, de acordo com cálculo amostral e critérios para realização do estudo. Foi aplicado como instrumento de coleta de dados um questionário com quesitos sociodemográficos e interrogativas acerca do climatério. De acordo com os resultados, a maioria das entrevistadas são casadas (56\%), com ensino médio (57\%), donas do lar (37\%) e 50\% informaram ser católicas. Em referência ao climatério, $63 \%$ informaram possuir conhecimento sobre o tema, $77 \%$ informaram que o climatério não é doença embora $73 \%$ acreditam que os sintomas não podem ser evitados. Verificou-se um percentual significativo de mulheres climatéricas em atendimento em unidades básicas de saúde e que essas mulheres não têm clareza a respeito das modificações e necessidade associadas à fase.

PALAVRAS-CHAVE: Saúde da Mulher. Climatério. Promoção da Saúde. Enfermagem.

Enfermeira. Mestranda. Mestrado Profissional em Saúde da Família, Faculdade de Enfermagem Nova I Esperança, FACENE. CEP: 58057-032, João Pessoa, Paraíba, Brasil. ORCID ID: 0000-0002-0386-829X; 0000-0001-5532-5580.

Enfermeira, Doutora em Enfermagem. Mestrado Profissional em Saúde da Família, Faculdade de Enfermagem Nova Esperança, FACENE. *Autor correspondente: vagna.cristinapb@gmail.com.

CEP: 58057-032, João Pessoa, Paraíba, Brasil. ORCID ID: 0000-0001-5649-8256; 0000-0002-8831-3620. 


\section{INTRODUÇÃO}

Segundo dados da Pesquisa Nacional por Amostra de Domicílios Contínua, realizada em 2019, as mulheres representam a maioria da população brasileira, cerca de $51,8 \%$ do contingente populacional é constituído por pessoas do sexo feminino. Na atualidade, a mulher tem importante representatividade social e diferentes aspectos passaram a ser identificados entre pessoas desse grupo, por exemplo, elas passaram a viver mais, ter menos filhos e ocupar cada vez mais espaço no mercado de trabalho ${ }^{1}$. Além dos importantes cargos no mercado de trabalho, são elas detentoras de maior sobrecarga das atividades domésticas, assumindo socialmente o papel de principal cuidador da família, aspectos que têm comprometido seu processo saúde doença. $^{2}$

Diversos eventos balizam o processo de vida das mulheres, suas trajetórias são marcadas por inúmeras mudanças biológicas, favorecendo a manifestação de algumas doenças que estão associadas, sejam elas clínicas ou endócrinas, fatores esses que podem tornar as mulheres vulneráveis aos mais diversos tipos de agravos, sejam de ordem psicológicas ou metabólicas. Entre eles, destaca-se o climatério, descrito como mudança da fase reprodutiva para não reprodutiva. Normalmente essa fase fisiológica tem início aos 40 anos de idade e prossegue até os 65 . Um tempo representativo na vida da mulher que carece de uma assistência de qualidade, como forma de contribuir na condução desse processo fisiológico, que normalmente demanda intervenções especializadas. 3

Em virtude do crescimento do número de doenças de ordem endócrinas, entre as mulheres, foi produzido o Manual de Atenção Integral à Saúde da Mulher no Climatério e
Menopausa elaborado pela Área Técnica, vinculada ao Ministério da Saúde, com o objetivo de concretizar as ações da Política Nacional de Atenção Integral à Saúde da Mulher, buscando promover qualidade na atenção às mulheres nessa fase da vida. Nesse manual estão inseridas diretrizes e princípios que orientam os cuidados de forma integral e humanizada, considerando as diversidades e especificidades desse grupo social. 4

Tendo em vista a grandiosidade de peculiaridades imbuidas no climatério, alguns estudos têm mostrado que as mulheres pouco sabem sobre essa fase. Na maioria dos casos, esse entendimento está restrito apenas as alterações físicas que acontecem nessa fase da vida, abrindo lacunas de conhecimento sobre possíveis alterações psicossociais que podem ser vivenciadas no período. 5,6

Cada pessoa vivencia de forma particular e variável os sintomas do climatério. É possível que ocorram sintomas neurovegetativos que estão associados a fase, ${ }^{6}$ além de diversas manifestações em reposta ao preconceito e tabus erigidos pela sociedade, ao longo dos tempos, podendo resultar em prejuízos pessoais e implicações sociais de grande relevância, tornando-as inseguras e vulneráveis a danos físicos e emocionais que afetam a qualidade de vida do grupo. 7

Enfatizando a necessidade de acompanhamento para mulheres no climatério, é essencial que ocorra o acolhimento e assistência adequada através da Estratégia Saúde da Família (ESF), por meio de profissionais capacitados que executem práticas clínicas de forma holística, atentandose para uma escuta qualificada, o incentivo ao autocuidado e a participação delas no seu processo saúde-doença. 8 
Na participação desse cenário clínico, se enfatiza a importância da atuação do profissional de Enfermagem, que investe diretamente no processo do cuidado apoiando a mulher. Faz-se necessário que esse profissional disponibilize ações que objetivem torná-las ativas no processo de cuidado para que possam compreender melhor as fases do climatério. É importante que o enfermeiro atue no planejamento da assistência de acordo com as necessidades de cada mulher,

\section{MATERIAL E MÉTODOS}

Trata-se de uma pesquisa descritiva, com abordagem quantitativa, realizada na Unidade Integrada de Estratégia Saúde da Família Nova União, que dispõe de 4 unidades de saúde: Prossind 1, Mangabeira 4, Panorâmica e União, fazendo parte do Distrito Sanitário II, João Pessoa/PB, no período de setembro a novembro de 2018. A escolha da referida unidade ocorreu pelo número expressivo de mulheres na fase climatérica assistidas nestes serviços.

Como critério de inclusão, foi delimitada a faixa etária de 45 a 65 anos, de acordo com os parâmetros estabelecidos pela Organização Mundial de Saúde (OMS). A partir desta delimitação, foi verificado como população do estudo 894 mulheres em período de climatério. Para definir a amostra foi realizado um cálculo com o samplesize do programa OPEN EPI (Open Source Statistics for Public Health) versão 2.3.1, considerando nível de confiança de $95 \%$ e margem de erro de 5\%, totalizando 169 mulheres a serem priorizando o cuidado humanizado e com qualidade. 9

Considerando a efetivação de programas específicos para esse grupo em serviços da atenção básica e que a mulher é figura cativa nestes serviços, este estudo tem como objetivo descrever o perfil sociodemográfico de mulheres climatéricas atendidas na Estratégia Saúde da Família e constatar o conhecimento das entrevistadas a respeito da fase climatérica. investigadas. No entanto, o alcance amostral ocorreu por conveniência e, considerando o período restrito da coleta de dados, não foi possível abordar o universo estimado, finalizando com uma amostra finita de 100 mulheres.

Para a coleta de dados, foi aplicado um formulário elaborado pelo pesquisador responsável, aplicado em sala reservada na unidade de saúde integrada, em dias úteis, turnos manhã e tarde, antes ou após a consulta em saúde.

Os resultados numéricos foram tratados e agrupados, para na sequência serem apresentados por meio da estatística descritiva e observar a distribuição de frequências das variáveis estudadas.

Este estudo foi aprovado pelo Comitê de Ética em Pesquisa da Faculdade de Enfermagem e Medicina Nova Esperança - FACENE/FAMENE, cujo número do CAEE é: 98808718.3.0.0000.5179. 


\section{RESULTADOS E DISCUSSÃO}

De acordo com os dados apresentados na Tabela 1, foi identificada heterogeneidade entre as características das usuárias do serviço de saúde. Em referência ao estado civil 56\% das participantes são casadas; no que se refere ao grau de escolaridade, houve predominância entre as investigadas, $57 \%$ afirmaram ter estudado até o ensino médio; em relação a ocupação, $37 \%$ delas afirmam ser cuidadoras do lar; no tocante a renda mensal, $28 \%$ relataram receber mais de um salário-mínimo; e, quanto a crença religiosa predominante entre o grupo foi católica, com $50 \%$.

TABELA 1: Perfil social das mulheres climatéricas cadastradas na Unidade Integrada de Estratégia Saúde da Família Nova União. João Pessoa, 2018.

\begin{tabular}{cccc}
\hline \hline Usuárias & Variáveis & $\mathbf{n}$ & $\%$ \\
\hline \multirow{3}{*}{ Estado civil } & Casada & 56 & 56 \\
& Solteira & 24 & 24 \\
& Divorciada/viúva & 17 & 17 \\
& Não respondeu & 3 & 3 \\
\hline \multirow{3}{*}{ Grau de escolaridade } & Fundamental & 23 & 23 \\
& Médio & 57 & 57 \\
& Superior & 20 & 20 \\
\hline \multirow{3}{*}{ Profissão } & Do lar & 37 & 37 \\
& ACS & 9 & 9 \\
& Serviços gerais & 8 & 8 \\
& Autônomas & 8 & 8 \\
& Téc. Enf. & 5 & 5 \\
Renda mensal & Outras & 33 & 33 \\
\hline & Mais de um salário & 28 & 28 \\
& Um salário & 5 & 5 \\
& Menos de um salário & 17 & 17 \\
& Em branco & 50 & 50 \\
\hline \multirow{2}{*}{ Religião } & Católica & 50 & 50 \\
& Evangélica & 40 & 40 \\
& Outras & 6 & 6 \\
& Em branco & 4 & 4 \\
\hline \hline TOTAL & & 100 & 100 \\
\hline \hline
\end{tabular}

Apresentar o perfil de um grupo de pesquisa possibilita conhecer a relação entre as características sociodemográficas e o fenômeno investigado, sendo assim faz-se imprescindível conhecer o perfil das mulheres climatéricas cadastradas em serviços da atenção básica e a influência dessas variáveis no processo saúde-doença.

No que se refere ao estado civil, verifica-se que a convivência com companheiro contribui de forma positiva no climatério. Nessa fase, a mulher demanda cuidados que precisam ser percebidos e entendidos, não só pela mulher, mas por quem convive ao seu redor. A família se torna influente e participante do processo de envelhecimento feminino transformando a passagem dessa fase mais agradável. O núcleo familiar é o espaço que elas compartilham as suas experiências e amenizam a ansiedade em decorrência das mudanças do corpo. Em contrapartida, a falta da escuta por parte dos cônjuges pode causar embates. Os companheiros são imprescindíveis para amenizar mal-entendidos, colaboram para melhor compreensão sobre as mudanças ocorridas no climatério, dão o suporte emocional fundamental para melhorar a qualidade de seus vínculos conjugais. ${ }^{10} \mathrm{Em}$ contrapartida, vale ressaltar que mulheres 
sem filhos nessa fase tendem a apresentar maior independência e maior autonomia profissional, aspecto que contribui positivamente no processo. ${ }^{11}$

Outro aspecto positivo identificado é a escolaridade. Identifica-se que a maioria das investigadas concluiu o ensino médio. $O$ grau de escolaridade é um determinante de impacto específico para qualidade de vida nos diferentes ciclos vitais da mulher. O nível de conhecimento favorável se faz de extrema importância, tornando-se um aliado essencial para qualidade de vida da população. É sabido que a baixa escolaridade pode comprometer a participação da mulher em atividades que beneficiam sua condição de saúde e como elas irão reagir diante das modificações decorrentes desse período, assim o nível de conhecimento contribui positivamente para qualidade de vida ao longo desse período. ${ }^{12}$

Diante do exposto, é de suma importância que os profissionais que estão à frente do atendimento utilizem estratégias educativas e suporte emocional para favorecer a participação da mulher na troca de conhecimento, abordando de forma adequada o climatério, a promoção da saúde, o tratamento imediato aos agravos e prevenção de possíveis danos. ${ }^{13}$

No que se refere a variável ocupação, verifica-se heterogeneidade nos resultados, apresentando a nova realidade da mulher no espaço social. Embora o percentual predominante seja de mulheres do lar, na totalidade essas mulheres têm ocupado diferentes espaços sociais pela sua inserção no mercado de trabalho. A participação da mulher na sociedade moderna exige dela a capacidade de administrar carreira profissional, o lar e a família, concentrando toda responsabilidade em si, o que consequentemente exerce grande impacto sobre a qualidade de vida delas. Ao exercer tais papéis, muitas mulheres se privam de realizar o próprio cuidado deixando de lado seu bem estar. 6

Um ponto positivo a ser destacado a respeito do exercício das atividades profissionais fora do lar é que essas atividades proporcionam a oportunidade de diálogo com outras pessoas, aspecto que contribui de forma positiva para a troca de informação, possibilitando a adoção de novas posturas, libertando-se de mitos e crenças, resultando em qualidade de vida na fase climatérica. ${ }^{14}$

A variável religiosidade também pode influenciar nas atitudes e qualidade de vida das mulheres climatéricas. Os dogmas religiosos comprometem comportamentos e posturas da mulher no contexto familiar e social. De acordo com os resultados apresentados, o catolicismo é uma das religiões que mais tem adeptos, precedida da religião evangélica que também cresce no contexto brasileiro. Partindo dessa premissa, quando a mulher está intimamente ligada as questões religiosas ela pode se tornar inibida para buscar apoio fora do lar, principalmente para trabalhar questões de sua intimidade relacionadas ao corpo e a sexualidade. Na maioria das vezes, esse grupo manifesta sentimentos de vergonha e acredita que algumas posturas são remetidas ao pecado. ${ }^{13}$

Independente dos períodos de vida a mulher necessita ter pleno acesso aos serviços de saúde e carece de um olhar diferenciado, especialmente nessa fase. Nesse contexto, a Atenção Básica é adequada para atender grande parte de suas necessidades, para isso é preciso que a rede esteja organizada de maneira que possa oferecer atendimento especializado para fase climatérica. 4

As mudanças na vida da mulher nesse período ultrapassam a sintomatologia clínica. Apesar de experienciar os vários sinais e sintomas, elas desconhecem ou não sabem reconhecer as alterações hormonais, fisiológicas e emocionais incluídas nas etapas do declínio da produção hormonal e na 
TABELA 2: Perfil social das mulheres climatéricas cadastradas na Unidade Integrada de Estratégia Saúde da Família Nova União. João Pessoa, 2018.

\begin{tabular}{cccc}
\hline Usuárias & Variáveis & $\mathbf{n}$ & $\%$ \\
\hline \hline \multirow{2}{*}{ Ouviu falar climatério } & sim & 63 & 63 \\
& não & 37 & 37 \\
\hline \hline \multirow{2}{*}{ Climatério é doença } & sim & 23 & 23 \\
& não & 77 & 77 \\
\hline Os sintomas podem ser & sim & 27 & 27 \\
evitados & não & 73 & 73 \\
\hline Mulheres no climatério & sim & 16 & 16 \\
precisam de tratamento & não & 84 & 84 \\
\hline \hline TOTAL & & 100 & 100 \\
\hline \hline
\end{tabular}

interrupção de ciclos menstruais. ${ }^{15}$

Ainda que os programas de saúde da mulher estejam em pauta desde o início do século $X X$, atualmente, verifica-se que $O$ enfoque dos programas e da assistência esteja direcionado a problemáticas específicas, a exemplo do câncer de mama, de colo de útero ou questões ligadas a gravidez. Dessa forma, faz-se necessário elucidar a importância de enfatizar ações direcionadas a suprir outras demandas femininas, a exemplo de questões relacionadas a fase do climatério e menopausa. ${ }^{16-17}$

Na tabela 2 são apresentados dados acerca do conhecimento das mulheres sobre o climatério. A maior parte $(63 \%)$ das investigadas relataram deter algum tipo de saber sobre o tema, $77 \%$ delas informaram que o climatério não é doença, $73 \%$ delas acreditam que os sintomas não podem ser evitados e $84 \%$ das entrevistadas acreditam que não existe a necessidade de tratamento para alívio das alterações que se manifestam.

É de extrema importância identificar quais conhecimentos as mulheres possuem sobre as questões relacionadas ao processo saúde-doença. A maioria das investigadas referiram conhecimento prévio a respeito do climatério. Ainda que muitas mulheres informem conhecimentos a respeito dessa temática, verifica-se que essas informações são superficiais e imersas por lacunas. Grande parte das vezes elas resguardam a visão de que o climatério é a chegada da terceira idade, da doença, da mudança hormonal ou se resume a um momento que faz parte da vida. ${ }^{15}$

O climatério não é apenas uma mudança do período reprodutivo para o não reprodutivo, pode ser compreendido por um conjunto de expressões biopsicossociais e hormonais. A sua adaptação é uma fase difícil, acompanhada de sofrimento físico e emocional e existe uma variedade de mulheres que estão mal informadas e susceptíveis a rejeição de si própria, à medida que começam a envelhecer e seu corpo passa por mudanças e, consequentemente, sofrem alterações nas suas relações pessoais, o que pode gerar dificuldades na sua vida. Em virtude disso, é de fato necessário que haja o desenvolvimento de ações educativas, com o intuito de enriquecer o conhecimento a respeito do tema para que possa transpor com dignidade esta fase. ${ }^{18}$

A maioria das participantes desse estudo referem que o climatério não é doença, vale salientar que estas questões estão ligadas diretamente as suas crenças culturais e que pode interferir tanto positivamente quanto negativamente na qualidade de vida. O climatério não deve ser compreendido enquanto doença e não é exigido um tratamento específico para essa fase. No entanto, quando sinais e sintomas se tornam evidentes, devem ser aliviados e prioritariamente é preciso que se faça uma abordagem educacional, de forma que a mulher se torne consciente dos efeitos que comumente são manifestados nessa fase. ${ }^{19}$ 
Considerando que esse estudo foi realizado com mulheres em atendimento em serviço da atenção básica, identificam-se fragilidades na assistência a essa demanda. Quando foi inquerido sobre a manifestação de sintomas indesejados a maioria respondeu que estes não podem ser evitados. Sabe-se que o climatério é um período no qual ocorrem consideráveis mudanças endócrinas, clínicas e metabólicas que não podem ser freadas, mas quando se verifica prejuízos e desconforto para o cotidiano da mulher ela deve estar orientada a procurar ajuda em serviços de saúde. As transformações dessa fase estão relacionadas a perda da função folicular ovariana, que contribui para o surgimento dos sintomas vasomotores, alterações urogenitais, alterações lipoprotéicas. Além desses fatores, pode ocasionar mudanças emocionais, a exemplo de depressão, dificuldades cognitivas e ansiedade, que são oriundas do baixo nível hormonal e que podem demandar intervenção apropriada. 20

Ainda sobre a sintomatologia, na maioria das vezes, está associada às manifestações climatéricas causadas pelo hipostrogenismo, que é a baixa dos níveis ideais de estrogênio no corpo, predominando sintomas vasomotores, referidos como ondas de calor, taquicardia, sudorese e atrofia urogenital, estado de ressecamento vaginal e dispareunia. Frequentemente ainda são relatadas pelas mulheres: mudanças de humor, perda da libido, dificuldades de memória, insônia e dores nas articulações. Estes sintomas, geralmente, estão relacionados a fatores psicológicos e ao estilo de vida adotado por elas. ${ }^{21}$

Em referência ao tratamento, a maioria das participantes ( $84 \%$ ) referem que não é preciso nenhum tipo de tratamento para os sintomas climatéricos. Diante desse fato, muitas mulheres que estão vivendo esse período não buscam por atendimento para aliviar desconfortos característicos e não realizam nenhum tipo de tratamento. Porém, existem vários métodos terapêuticos que são eficazes no enfrentamento e alívio da sintomatologia climatérica, tais como prática de atividade física de forma regular, terapia de reposição hormonal, homeopatia, acupuntura, uso de fitoterapia e alimentação saudável, orientações e encaminhamentos que deveriam acessar em serviços da atenção básica. $^{22}$

Perante o exposto, torna-se de extrema importância que os profissionais de enfermagem que estão à frente do atendimento básico de saúde, proporcionem a essas mulheres estratégias que possibilitem maiores conhecimento sobre o climatério, para promoção da saúde, acesso ao tratamento imediato, adotando ações como foco para prevenção de possíveis danos pelo não acesso as estratégias terapêuticas quando necessário. Por se tratar de cuidados permeados de particularidades, faz-se necessárias ações multiprofissionais em serviços da atenção básica, normalmente nesses serviços o enfermeiro é interlocutor dessas ações. 23,24

$O$ enfermeiro deve traçar metas de cuidados que estejam relacionadas e direcionadas as características sociodemográficas do grupo alvo, a sintomatologia do climatério, pois cada mulher manifesta de forma singular os sintomas. Assim, as ações precisam ser planejadas de maneira que atendam cada uma de suas necessidades, visando o alívio das manifestações do período. O profissional deve estar preparado para detectar essas manifestações e tentar minimizar os efeitos. É fundamental que o plano de assistência seja adequado a realidade de cada mulher. Portanto, o acolhimento e o diálogo são algumas das estratégias adotadas para esclarecer as dúvidas e orientar sobre as possíveis mudanças físicas e psicológicas 
advindas da fase. ${ }^{24}$

Posto isto, a assistência prestada na Estratégia Saúde da Família, deve priorizar o acolhimento, visto que este é considerado uma estratégia organizada do processo de trabalho da equipe de profissionais que centram suas atividades no usuário, tendo como base a escuta qualificada das suas necessidades. Dessa forma, a relação entre profissional e usuária se estreita através do estabelecimento de uma convivência de confiança. Isso se dá pela realização de uma escuta ativa, do diálogo, da empatia, do respeito, possibilitando uma visão integral do outro, fatores que são fundamentais e garantem a humanização e a qualidade do atendimento. 25

Faz-se imprescindível uma atenção especial por parte de profissionais da atenção básica às mulheres que vivenciam o período do climatério como forma de identificar e suprir as demandas psíquicas e orgânicas que se manifestam na fase.

\section{CONCLUSÃO}

Nesse estudo, verifica-se um percentual significativo de mulheres climatéricas em atendimento em serviços da atenção básica, e de acordo com o perfil, identifica-se uma heterogeneidade do grupo com características positivas que contribuem para ações educativas e interventivas para suprir as necessidades apresentadas nessa fase.

De acordo com os resultados desse estudo, verifica-se que na maioria das vezes as mulheres não têm clareza a respeito das modificações e necessidades associadas à fase do climatério e que a maior parte das investigadas desconheciam possibilidades de tratamento para aliviar os sintomas e evitar maiores danos a sua saúde, embora demonstrassem o conhecimento sobre alguns dos sintomas vivenciados.

Independente da fase, a mulher carece de acesso livre aos serviços de saúde e as informações, para se tornarem ativas no seu processo saúde-doença e corresponsável pelo seu bem-estar e qualidade de vida. Assim, a mulher deve ser acolhida de forma qualificada e humanizada, considerando a qualidade dos serviços prestados e a resolutividade das suas necessidades. Faz-se necessário promoção de cuidados com mais ações específicas direcionadas a mulher climatérica, valorizando ações de prevenção, promoção e tratamento em serviços da atenção básica.

\section{KNOWLEDGE OF ASSISTED WOMEN IN THE FAMILY HEALTH STRATEGY REGARDING THE CLIMACTERIC}

\section{ABSTRACT}

Important events mark the life process of women, they face organic and psychic changes related to 
vital cycles, including climacteric. The aim of this study is to describe the socio demographic profile of climacteric women assisted in the Family Health Strategy and to verify the interviewees' knowledge about the climacteric phase. The research is descriptive with quantitative approach, carried out in a health unit in the city of João Pessoa- PB. The population consisted of 894 women, during the climacteric period registered in the unit. A sample of 100 women was defined according to sample calculation and criteria for the study. A questionnaire was applied as a data collection instrument with sociodemographic and interrogative questions about the climacteric. According to the results, most of the interviewees are married (56\%), with high school education (57\%), owners of the home $(37 \%)$ and $50 \%$ reported being Catholic. In reference to the climacteric, $63 \%$ reported having knowledge about the subject, $77 \%$ reported that climacteric is not a disease although $73 \%$ believe that symptoms cannot be avoided. There was a significant percentage of climacteric women in primary health care units and that these women do not have clarity about the changes and needs associated with the phase.

KEYWORDS: Women's Health. Climacteric. Health Promotion.

\section{REFERÊNCIAS}

1. Instituto Brasileiro de Geografia e Estatística. IBGE Educa Jovens. Conheça o Brasil-População: quantidade de homens e mulheres, 2019.

2. Carvalho, EB. Uso do tempo em cuidadores familiares de idosos com demências. [Tese de doutorado em Gerontologia]. Campinas - SP: Faculdade de Ciências Médicas da Universidade Estadual de Campinas; 2017. 93p.

3. Souza SS, Santos RL, Santos ADF, Barbosa MO, Lemos ICS, Machado MFAS. Mulher e climatério: concepções de usuárias de uma unidade básica de saúde. Reprod clim. 2017; 32(2): 85-89.

4. Ministério da Saúde (BR). Secretaria de Atenção à Saúde. Departamento de Ações Programáticas Estratégicas. Manual de Atenção à Mulher no Climatério. Brasília - DF. Editora do Ministério da Saúde, 2008.192 p.

5. Leite TAS, Nunes JSS, Pereira AJ, Silva ML. Conhecimento de mulheres jovens sobre a menopausa e sintomas climatéricos. Braz. J. Hea. 2020; 3(3): 7204-12.

6. Nascimento SS. Mulheres no climatério: qualidade de vida em seu cotidiano. [Trabalho de
Conclusão de Curso em Enfermagem]. Campina Grande: Universidade Federal de Campina Grande; 2016. 56p.

7. Patrício RSO, Ribeiro Júnior OC, Ferreira SMS, Araújo TS, Brasil LC, Silva JM et al. Ações de enfermagem na promoção da saúde e qualidade de vida de mulheres no climatério. REAEnf/EJNC. 2020; 4: e 4782.

8. Vieira TMM, Araujo CR, Souza ECS, Costa MAR, Teston EF, Benedetti GMS et al. Vivenciando o climatério: percepções e vivências de mulheres atendidas na atenção básica. Enferm. Foco 2018; 9 (2): 40-45.

9. Curta JC, Weissheimer AM. Percepções e sentimentos sobre as alterações corporais de mulheres climatéricas. Rev Gaúcha Enferm. 2020; 41. 14p. (esp): e20190198.

10. Silva VH, Rocha JSB, Caldeira AP. Fatores associados à autopercepção negativa de saúde em mulheres climatéricas. Ciênc. Saúde Coletiva [online]. 2018; 23(5): 1611-20.

11. Mazzetto FMC, Ferreira MLSM, Marim MJS, Orso LF. Os sentidos do climatério para mulheres 
sem filhos. Atas-Investigação Qualitativa em Saúde. 2017; 2: 347-55.

12. Morais FC, Luiz LNS. Integralidade na assistência à saúde da mulher no climatério. [Trabalho de Conclusão de Curso em Enfermagem]. Goiás: Centro Universitário de Anápolis Unievangélica; 2018, 32p.

13. Alcântara LL, Nascimento LC, Oliveira VAC. Conhecimento das mulheres e dos homens referente ao climatério e menopausa. Enferm. Foco. 2020; 11 (1): 44-49.

14. Benetti IC, Sales LS, Junior João PR, Deon APR, Wilhelm FA. Climatério, enfrentamento e repercussões no contexto de trabalho: vozes do Extremo Norte do Brasil. Revista KairósGerontologia, São Paulo - SP. 2019; 22(1): 123-46.

15. Fonseca JR, Freitas RF, Duarte MR, Reis VMCP, Popoff DAV, Rocha JSB. Índice de Massa Corporal e fatores associados em mulheres climatéricas. Enfermería Global. $\mathrm{N}^{\circ}$ 24. Janeiro, 2018.

16. Alencar YLR. Conhecimentos e vivências de mulheres acerca do climatério: uma revisão integrativa. [Trabalho de Conclusão de Curso em Enfermagem]. Campina Grande: Universidade Federal de Campina Grande; 2019. 48p.

17. Tavares VMC, Neto LMA, Pereira ES, Taveira MGMM, Cavalcante JK, Correia DS. Roda de conversa: atenção integral à saúde das mulheres e questões de gênero. Brazilian Journal of Development. 2020; 6(8): 61501-10.

18. Carneiro VSM, Adjuto RNP, Alves KAP. Saúde do homem: identificação e análise dos fatores relacionados à procura, ou não, dos serviços de atenção primária. Arq. Ciênc. Saúde UNIPAR, Umuarama. 2019; 23(1): p. 35-40.

19. Rezende FCB, Lisboa HKS, Almeida LAV, Lima
ER, Souza MS, Barbosa RAA et al. A sexualidade da mulher no climatério. Revista da Universidade Vale do Rio Verde. 2019; 17(1): 1-10.

20. Oliveira ZM. (Re)significando o climatério de mulheres que o vivenciam na perspectiva do interacionismo simbólico. [Tese de doutorado em Enfermagem]. Rio de Janeiro: Universidade do estado do Rio de Janeiro; 2019.

21. Dias PA. Associação do nível de atividade física com os sintomas climatéricos, ansiedade e depressão em mulheres no climatério. [Trabalho de conclusão do curso de Educação Física]. Uberlândia: universidade Federal de Uberlândia; 2018.

22. Pedro AO, Pinto-Neto AM, Costa-Paiva LHS, Osis MJD, Hardy EE. Síndrome do climatério: inquérito populacional domiciliar em Campinas, SP. Rev Saúde Pública 2003; 37(6): 735-42.

23. Freire AL, Araújo KS, Vila $A C D$, Araújo, MAS. Assistência de enfermagem à mulher no climatério e sua sexualidade: relato de experiência na atenção básica. Revista eletrônica de trabalhos acadêmicos - Universo/Goiânia volume 1, $\mathrm{n}^{\circ} 1,2016$.

24. Melo AAC, Silva EPC, Giotto AC. Assistência da enfermagem à mulher no climatério na atenção básica de saúde. Rev Inic Cient e Ext. 2019; 2(4): 213-08.

25. Andrade DBS, Lira FNA, Silva EV, Aoyama EA, Farias FC. O papel do enfermeiro nos cuidados de enfermagem com mulheres no período climatérico. Rev. Cient. Sena Aires. 2018; 7(1): 1822.

26. Silva MTA, Silva Júnior VB, Mangueira JO, Melo APL. Implantar o acolhimento na estratégia de saúde da família é viável? Em Extensão, Uberlândia. 2018; 17(1): 153-65. 